\title{
Randomized Comparison of the Continuous vs Point- by-Point Radiofrequency Ablation of the Cavotricuspid Isthmus for Atrial Flutter
}

\author{
Shinsuke Miyazaki, MD; Atsushi Takahashi, MD; Taishi Kuwahara, MD; Atsushi Kobori, MD; \\ Yasuhiro Yokoyama, MD; Toshihiro Nozato, MD; Akira Sato, MD; \\ Kazutaka Aonuma, MD*; Kenzo Hirao, MD**; Mitsuaki Isobe, MD**
}

\begin{abstract}
Background Achievement of complete conduction block in the cavotricuspid isthmus (CTI) is a curative ablation technique in patients with common atrial flutter (AFL). The present study was a prospective comparison of the efficacy of 2 ablation strategies in patients with common AFL: the continuous and point-by-point radiofrequency (RF) delivery techniques.

Methods and Results Forty patients with common AFL were randomly assigned to either a group treated with a continuous RF delivery or to a group undergoing point-by-point RF ablation. In the first group, the RF energy was continuously delivered during a slow drag of the catheter tip from the tricuspid annulus to the inferior vena cava without stopping the application. In the second group, the RF ablation was performed using a point-by-point approach for $60 \mathrm{~s}$ at each point. All patients underwent ablation with an 8-mm-tip ablation catheter with a power limit of $50 \mathrm{~W}$ and a target temperature of $55^{\circ} \mathrm{C}$. Complete CTI conduction block was achieved in all patients. The patient characteristics, including the anatomy of the CTI estimated by 3-dimensional computed tomography, were no different between the 2 groups. The procedure time (time from the start of RF delivery to the completion of CTI block), fluoroscopic time and total RF energy required to create the CTI block between the continuous and point-by-point groups were $7.3 \pm 5.6$ vs $21.2 \pm 22.2 \mathrm{~min}$ ( $\mathrm{p}<0.01), 7.2 \pm 4.4$ vs $16.2 \pm 14.1 \mathrm{~min}(\mathrm{p}<0.05)$, and $15,631 \pm 6,001$ vs $24,072 \pm 16,140$ joules $(\mathrm{p}<0.05$ ), respectively. There were no complications or recurrences of AFL during the follow-up period in any of the patients.

Conclusion In the curative treatment of common AFL, the continuous RF delivery approach could shorten the procedure and fluoroscopic time and reduce the total RF energy compared with the point-by-point RF ablation approach. (Circ J 2007; 71: 1922-1926)
\end{abstract}

Key Words: Atrial flutter; Catheter ablation; Cavotricuspid isthmus

C ommon atrial flutter (AFL) is a macroreentrant circuit around the tricuspid annulus (TA), and the transverse conduction block at the posterior right atrium is important for the maintenance of AFL! Radiofrequency (RF) ablation of the cavotricuspid isthmus (CTI), with complete bidirectional conduction block, is a highly effective treatment for this arrhythmia? ${ }^{2-4}$ Creating the CTI block involves continuous RF delivery during a slow drag of the catheter tip or by a succession of point-by-point applications from the TA to the inferior vena cava (IVC). Because it has not been fully elucidated which approach is more effective, we performed a prospective randomized comparison of these 2 approaches for the achievement of CTI bidirectional conduction block.

(Received June 11, 2007; revised manuscript received July 19, 2007; accepted July 31, 2007)

Cardiovascular Center, Yokosuka Kyosai Hospital, Yokosuka, *Department of Cardiology, University of Tsukuba Graduate School, Tsukuba and **Department of Cardiovascular Medicine, Tokyo Medical and Dental University, Tokyo, Japan

Mailing address: Shinsuke Miyazaki, MD, Cardiovascular Center, Yokosuka Kyosai Hospital, 1-16 Yonegahamadori, Yokosuka 2388558, Japan. E-mail: mshinsuke@k3.dion.ne.jp

\section{Methods}

Study Population

From May 2005 to April 2006, 48 patients were referred to Yokosuka Kyosai Hospital for RF ablation of paroxysmal AFL, 8 of whom had a history of catheter ablation for AFL or cardiac surgery and were excluded from this study. The remaining 40 patients were equally randomized to a continuous RF delivery group or a point-by-point group. The patient characteristics are shown in Table 1, and there was no difference noted between the 2 groups. All patients gave written informed consent before the procedure.

$R F$ Ablation of the CTI and Study Protocol

All antiarrhythmic drugs were discontinued more than

Table 1 Patients' Clinical Characteristics

\begin{tabular}{lccl}
\hline \hline & Point-by-point & Continuous & \\
\hline Age $($ years) & $58 \pm 10$ & $59 \pm 10$ & $N S$ \\
Gender $(F / M)$ & $18 / 2$ & $16 / 4$ & $N S$ \\
Structural heart disease & 4 & 3 & $N S$ \\
Ischemic heart disease & 2 & 2 & \\
Valvular heart disease & 1 & 1 & \\
Cardiomyopathy & 1 & 0 & \\
\hline
\end{tabular}


A

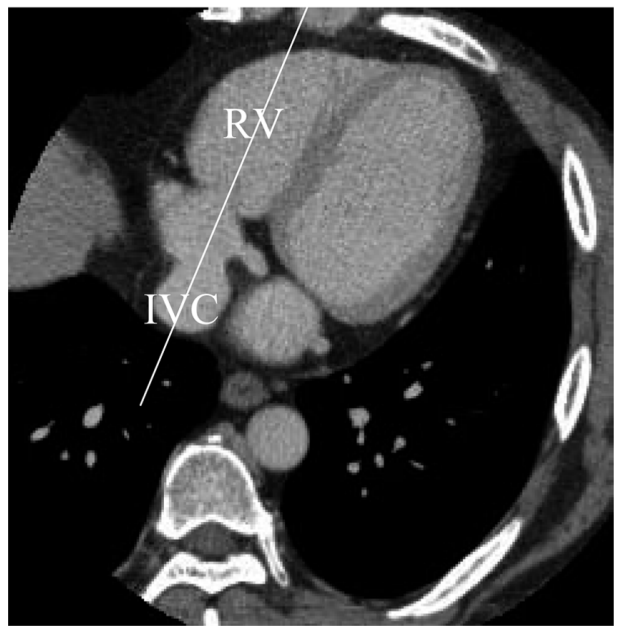

B

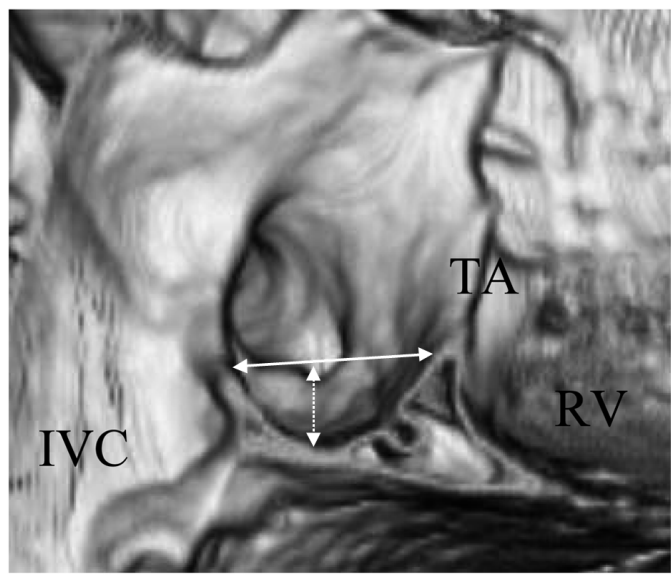

Fig 1. (A) Horizontal section of the cavotricuspid isthmus (CTI) on multidetector row computed tomography. The CTI image in vertical section was obtained along the line (white line) between the inferior vena cava (IVC) and lower hinge point of the tricuspid annulus (TA). (B) The length and depth of the CTI were respectively defined as the distance between the edges of the IVC and TA, and the perpendicular distance between the line and deepest point of the isthmus. RV, right ventricle.

7 days before the ablation procedure. All patients had had effective anticoagulation for $>1$ month, and transesophageal echocardiography was performed to exclude any atrial thrombi before the ablation?

The surface electrocardiogram (ECG) and bipolar intracardiac electrograms were continuously monitored and stored on a computer-based digital recorder system. The bipolar electrograms were filtered from 30 to $500 \mathrm{~Hz}$ and measured at a sweep speed of $100 \mathrm{~mm} / \mathrm{s}$. All measurements were performed with a Cardiolab system (Prucka Engineering). A pentapolar electrode catheter was advanced to the His bundle region, and 2 decapolar deflectable catheters were positioned around the TA and within the coronary sinus, respectively. RF current application was delivered in a unipolar mode between the distal 8-mm-tip of the ablation catheter (Japan Lifeline, Inc) and a cutaneous patch electrode placed over the left scapula during proximal coronary sinus pacing using temperature-controlled RF delivery with a power limit of $50 \mathrm{~W}$ and a target temperature of $55^{\circ} \mathrm{C}$.

In the patients randomized to the continuous group, the RF energy was continuously delivered during a slow drag of the catheter tip, drawn $4-6 \mathrm{~mm}$ each, from the TA to the IVC anatomically without stopping the RF delivery; the total drag time was 3-6 min. In the patients assigned to the point-by-point group, the RF delivery was applied at each point for $60 \mathrm{~s}$ and $30-60 \mathrm{~s}$ of RF cessation was set to find the next site after each ablation. The RF applications were started at the ventricular aspect of the TA when a stable electrogram with a small atrial and large ventricular potential was observed. The catheter was withdrawn after each application to find a sharp and large atrial potential, under fluoroscopic guidance, to produce a continuous lesion until the IVC was reached. When bidirectional block was not created in either group, RF application was electrophysiologically added at the site of the conduction gap?

The procedure endpoint was complete bidirectional CTI block, and was assessed by recording widely separated local double potentials along the ablation line during atrial pacing. During pacing from the coronary sinus ostium, a clockwise isthmus block was assumed when a craniocaudal
Table 2 Echocardiography and MDCT Parameters

\begin{tabular}{llll}
\hline \hline & Point-by-point & Continuous & \\
\hline Left atrial diameter $(\mathrm{mm})$ & & & \\
$\quad$ Parasternal & $40.2 \pm 5.1$ & $38.6 \pm 4.9$ & $\mathrm{NS}$ \\
$\quad$ Transverse & $57.6 \pm 10.4$ & $55.8 \pm 7.7$ & $\mathrm{NS}$ \\
$\quad$ Longitudinal & $41.6 \pm 4.3$ & $39.5 \pm 5.0$ & $\mathrm{NS}$ \\
Right atrial diameter $(\mathrm{mm})$ & & & \\
$\quad$ Transverse & $50.8 \pm 7.9$ & $49.8 \pm 6.2$ & $\mathrm{NS}$ \\
$\quad$ Longitudinal & $33.3 \pm 5.8$ & $35.0 \pm 6.7$ & $\mathrm{NS}$ \\
LVEF $(\%)$ & $66.0 \pm 6.4$ & $66.9 \pm 7.2$ & $\mathrm{NS}$ \\
LAAF $(\mathrm{cm} / \mathrm{s})$ & $62.1 \pm 29.4$ & $61.0 \pm 28.0$ & $\mathrm{NS}$ \\
IVC isthmus length $(\mathrm{mm})$ & $22.0 \pm 6.2$ & $21.8 \pm 6.4$ & $\mathrm{NS}$ \\
CTI depth $($ mm) & $3.56 \pm 2.6$ & $3.37 \pm 3.8$ & $\mathrm{NS}$ \\
\hline
\end{tabular}

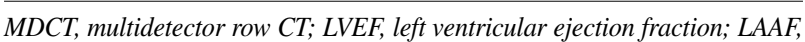
left atrial appendage flow; IVC, inferior vena cava; CTI, cavo-tricuspid isthmus.

activation sequence of the entire lateral right atrial wall was observed, and the second component of the local double potential recorded by the ablation catheter positioned over the ablation line was later than the potential recorded from the low lateral right atrium, and then during atrial pacing from the low lateral right atrium adjacent to the ablation line, a counterclockwise block was confirmed with a differential pacing method?

The procedure time, fluoroscopy time, and total RF energy required to create the CTI conduction block were calculated from the start of RF delivery to the completion of the bidirectional block. Bidirectional CTI block was confirmed again $30 \mathrm{~min}$ after the creation of the block line. After all the ablation procedures, coronary angiography was performed in all patients.

\section{Estimation of the CTI Anatomy Using 64-Slice Multidetector Row CT (MDCT)}

To estimate the differences in the isthmus anatomy between the 2 groups, we performed 64-slice MDCT 1-2 days before the ablation procedure. A contrast agent $(90 \mathrm{ml}$ of Iopamidol, Schering, Berlin, Germany) was injected intra- 


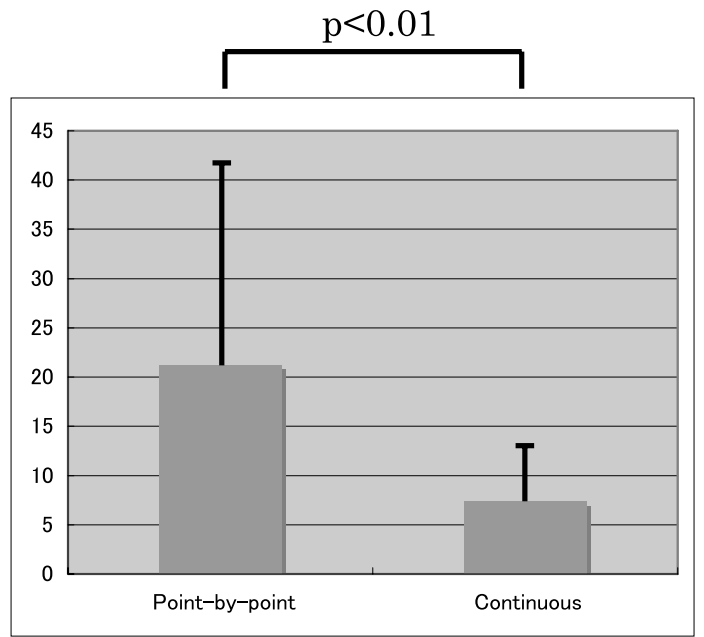

Procedure duration ( $\mathrm{min})$

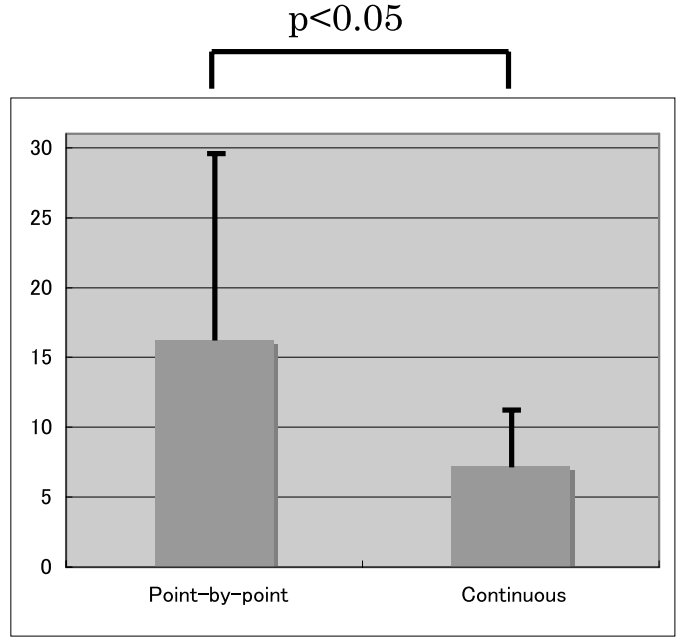

Fluoroscopic time (min)

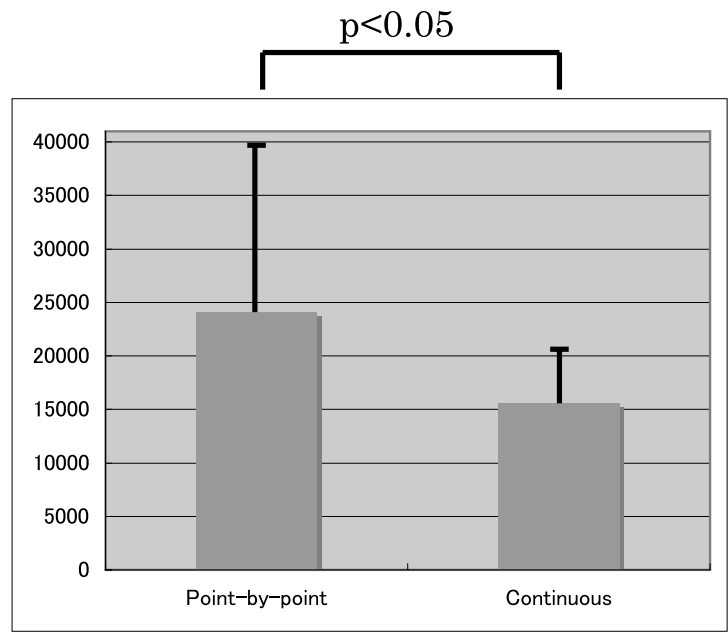

Total RF energy (J)

Fig 2. Procedural and fluoroscopic times were significantly shorter in the continuous group than in the point-by-point group, and a lower total radiofrequency (RF) energy was required to create the cavotricuspid isthmus block in the former.

venously at a flow rate of $3 \mathrm{ml} / \mathrm{s}$, and the scan was initiated $70 \mathrm{~s}$ after the beginning of the contrast agent injection during a single breath-hold. The image reconstruction was retrospectively gated to the ECG. The position of the reconstruction window was set at $75 \%$ of the RR interval. The MDCT data were transferred to an offline 3-dimensional image analysis workstation (ZIO M900, Amin, Tokyo, Japan).

The length of the CTI was obtained between the IVC and lower hinge point of the TA (Fig 1). The perpendicular distance between the line and deepest point of the isthmus, which was the depth of the CTI, was also measured.

\section{Echocardiography}

All patients underwent echocardiography to obtain the following data: left atrial diameter evaluated by the parasternal view, and longitudinal and transverse diameters of both atria measured by the 4-chamber view. The left atrial appendage flow was also assessed on the transesophageal echocardiogram.

\section{Follow-up}

After the procedure, no antiarrhythmic drugs were prescribed, and all patients were scheduled to visit the clinic and undergo 24-h Holter ECG at 12 and 24 weeks after their discharge. After 1 month, in the absence of any AFL recurrence, anticoagulant treatment was discontinued unless other major risk factors were present. A recurrence of AFL was defined according to the patient's symptoms, ECG and Holter ECG.

\section{Statistical Analysis}

The continuous variables are expressed as the mean $\pm \mathrm{SD}$. The continuous and categorical variables were compared with Student's t-test and chi-square test, respectively. A probability value of $\mathrm{p}<0.05$ indicated statistical significance.

\section{Results}

\section{Echocardiogram and MDCT}

The echocardiographic data and CT parameters are 
A
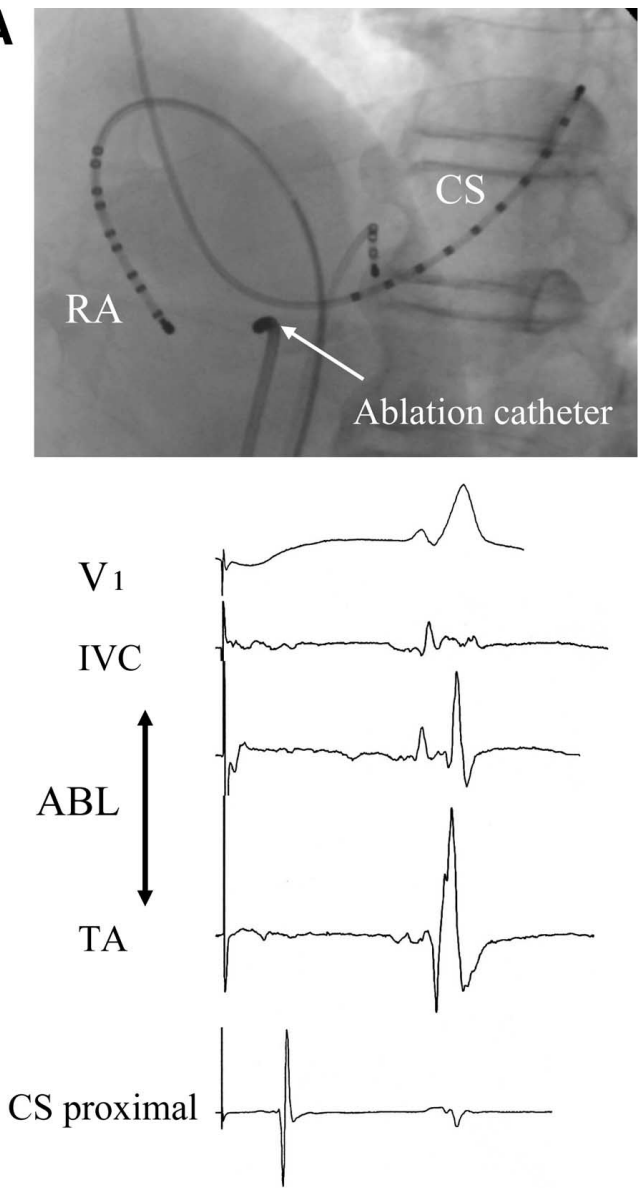

B

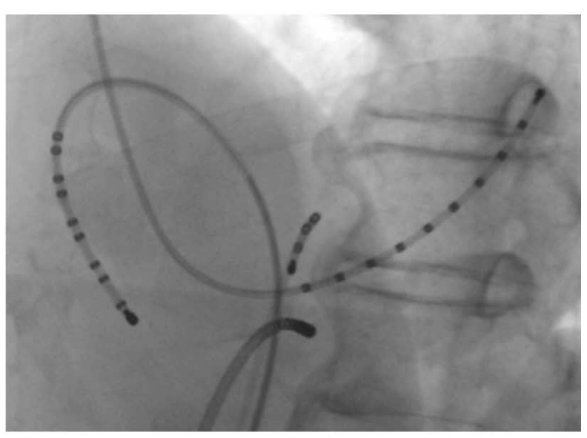

$\mathrm{V}_{1}$

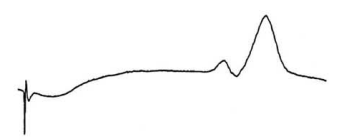

ABL

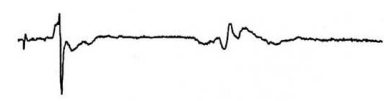

CS proximal

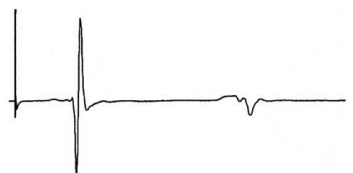

Fig 3. Representative withdrawal mapping in the cavotricuspid isthmus (CTI) after completion of the first ablation line using point-by-point radiofrequency (RF) application and the successful ablation site. Catheter position in the left anterior oblique (LAO) view. A pentapolar catheter in the His bundle region, 2 decapolar catheters along the right atrial (RA) lateral wall and in the coronary sinus (CS), and an ablation catheter on the CTI in the $06.00 \mathrm{~h}$ position of the LAO view. (A) Sequentially reconstituted withdrawal mapping from the tricuspid annulus (TA) to the inferior vena cava (IVC) during low lateral RA (LLRA) pacing after completion of the first ablation line shows the reduced atrial potentials, and no residual conduction gap can be seen along the previous ablation line. (B) Early and sharp atrial potentials during LLRA pacing at a site medial to the previous ablation line, and CTI block was achieved by an RF application at that site. $V_{1}$, surface electrocardiogram lead $\mathrm{V}_{1}, \mathrm{CS}$, bipolar electrogram recorded from the proximal pair of the CS catheter; ABL, bipolar electrogram recorded from the ablation catheter.

shown in Table 2. The left and right atrial chamber sizes and the length and depth of the CTI did not differ between the 2 groups.

\section{Comparison of the Ablative Parameters}

Successful CTI bidirectional conduction block was achieved in all patients. The comparison of the ablative parameters between the continuous and point-by-point groups is shown in Fig 2. The procedure and fluoroscopic times were significantly shorter in the continuous group than in the point-by-point group (procedure time: 7.3 \pm 5.6 vs $21.2 \pm 22.2 \mathrm{~min}, \mathrm{p}<0.01$, fluoroscopic time: $7.2 \pm 4.4$ vs $16.2 \pm 14.1 \mathrm{~min}, \mathrm{p}<0.05$ ), and a lower total RF energy was required to create the CTI block in the continuous group than in the point-by-point group $(15,631 \pm 6,001$ vs $24,072 \pm$ $16,140$ joules, $\mathrm{p}<0.05)$.

Five $(25 \%)$ patients in the continuous group and 15 (75\%) in the point-by-point group required additional RF applications to eliminate CTI conduction after the completion of the first ablation line $(\mathrm{p}<0.01)$. Residual conduction gaps were identified along the previous ablation line and were eliminated by additional RF applications in 5 patients in each group. In the remaining 10 patients of the point-bypoint group, no residual conduction gaps could be identified along the previous ablation line because of reduced atrial potentials (Fig 3A) and the RF applications placed along the ablation line were not effective. RF applications at sites just lateral or medial to the previous ablation line were required to achieve bidirectional CTI conduction block (Fig 3B).

There were no cases of pericardial effusion, tamponade, atrioventricular block or other major complications in either group. Coronary angiography performed after CTI ablation revealed normal coronary arteries in all patients.

During a follow-up period of 6 months, none of the patients had any recurrence of AFL after the ablation procedure.

\section{Discussion}

Major Findings

To the best of our knowledge, this study is the first pro- 
spective randomized trial to compare 2 approaches of $\mathrm{RF}$ delivery for creating CTI bidirectional block. The results of this study demonstrate that continuous RF delivery from the TA to the IVC could shorten the procedure and fluoroscopic times and requires lower RF energy to create the CTI block as compared with the point-by-point RF ablation.

\section{Mechanisms of the Superiority of Continuous Ablation}

$\mathrm{RF}$ ablation has been reported to produce edema around the ablative lesions ${ }^{8,9}$ In our study, the atrial potentials reduced and residual conduction gaps could not be identified along the previous ablation line after completion of the first ablation line in $50 \%$ of the point-by-point group patients. We speculate that these findings indicate the production of edema along the ablation line. In the point-by-point group we allowed 30-60 s for finding the next ablative site with a sharp and large atrial potential and good catheter stability and that time period may have contributed to the spread of the endocardial edema, leading to difficulty in making transmural myocardial lesions adjacent to the ablative lesions. However, the continuous ablation technique appeared to be able to create transmural lesions before any increase in edema occurred. The phenomenon of endocardial edema may protect the ablative energy from reaching the deeper layers of the myocardium, and could be the main reason for the difficulty with point-by-point ablation in creating CTI block as compared with continuous ablation. Additionally, if any conduction gaps remained in the epicardial myocardium, it might be difficult to find the electrical potentials because of the thickening of the endocardium with edema. In fact, the atrial potentials in the CTI became reduced and dull in half of the patients in the point-by-point group after the additional RF applications before the completion of CTI block, which resulted in difficulty in closing the electrical conduction gaps.

\section{Influence of CTI Morphology}

Previous anatomic and angiographic studies of human hearts have reported an anatomic variability in the isthmus, with a short or long length of the CTI and different morphologies! 10,11 The thickness of the myocardial tissue and length and morphology of the isthmus are proposed factors that influence the ablation procedure $!^{1-13}$ Costa et al reported that both a long CTI and concavity of the isthmus morphology were factors that could significantly increase the X-ray exposure duration and require more RF applications.$^{12}$ In this study, we did not find any differences in the length or depth of the CTI between the 2 groups.

\section{Safety of Continuous RF Ablation With an 8-mm-Tip Catheter}

Higher RF energies are also potentially associated with a higher risk of complications, including coronary artery injury and the pop phenomenon. Acute coronary occlusion secondary to RF ablation has been reported ${ }^{14}$ In the present study, all patients in each group underwent successful ablation of AFL without any complications and postprocedural coronary angiography revealed no coronary artery injuries.

\section{Study Limitations}

One of the limitations of this study is the small sample size. Also, MDCT could accurately show the anatomy of the CTI, but it was difficult to assess the transmural thickness of the myocardium in the isthmus. Therefore, we did not evaluate the difference in myocardial thickness between the 2 groups. In the point-by-point group, we set a time of $30-60$ s for finding the location of the next ablation site between each RF application and that may have accounted for the increased procedure time. However, the fluoroscopic time was definitely longer and more RF energy was required in that group. Difficulty in finding the conduction gaps along the ablation line seemed to be the main reason for the longer procedure time. Moreover, although 30-60s may not be needed to find the next ablation site, careful maneuvering of the ablation catheter takes time while searching for a good site.

\section{Conclusions}

In the ablation of the CTI in patients with common AFL, continuous RF delivery approach could shorten the procedure and fluoroscopic times, and require lower RF energy compared with the point-by-point ablation strategy.

\section{References}

1. Okumura Y, Watanabe I, Ashino S, Kofune M, Ohkubo K, Takagi Y, et al. Electrophysiologic and anatomical characteristics of the right atrial posterior wall in patients with and without atrial flutter. Circ J 2007; 71: 636-642.

2. Feld GK, Fleck RP, Chen PS, Boyce K, Bahuson TD, Stein JB, et al. Radiofrequency catheter ablation for the treatment of human type I atrial flutter: Identification of a critical zone in the reentrant circuit by endocardial mapping techniques. Circulation 1992; 86: 1233-1240.

3. Poty H, Saoudi N, Nair M, Anselme F, Letac B. Radiofrequency catheter ablation of atrial flutter: Further insights into the various type of isthmus block: Application to ablation during sinus rhythm. Circulation 1996; 94: 3204-3213.

4. Cosio FG, Lopez-Gil M, Goicolea A, Arribas F, Barroso JL. Radiofrequency ablation of the inferior vena cava-tricuspid valve isthmus in common atrial flutter. Am J Cardiol 1993; 71: 705-709.

5. Isobe N, Taniguchi K, Oshima S, Kamiyama H, Ezure M, Kaneko T, et al. Left atrial appendage outflow velocity is superior to conventional criteria for predicting of maintenance of sinus rhythm after simple cryoablation of pulmonary vein orifices. Circ J 2005; 69: 446-451.

6. Shah D, Haissaguerre M, Jais P, Fischer B, Takahashi A, Hocini M, et al. Simplified electrophysiologically directed catheter ablation of recurrent common atrial flutter. Circulation 1997; 96: 2505-2508.

7. Shah D, Haissaguerre M, Takahashi A, Jais P, Hocini M, Clementy J. Differential pacing for distinguishing block from persistent conduction through an ablation line. Circulation 2000; 102: 1517-1522.

8. Tanno K, Kobayashi Y, Kurano K, Kikushima S, Yazawa T, Baba T, et al. Histopathology of canine hearts subjected to catheter ablation using radiofrequency energy. Jpn Circ J 1994; 58: 123-135.

9. Tabuchi T, Okumura K, Matsunaga T, Tsunoda R, Jougasaki M, Yasue $\mathrm{H}$. Linear ablation of the isthmus between the inferior vena cava and tricuspid annulus for treatment of atrial flutter: A study in the canine atrial flutter model. Circulation 1995; 92: 1312-1319.

10. Cabrera JA, Quintana DS, Ho SY, Medina A, Wanguemert F, Gross $\mathrm{E}$, et al. Angiographic anatomy of the inferior right atrial isthmus in patients with and without history of common atrial flutter. Circulation 1999; 99: 3017-3023.

11. Heibuchel H, Willems R, Van Rensburg H, Adams J, Ector H, Van de Werf F. Right atrial angiographic evaluation of the posterior isthmus: Relevance for ablation of typical atrial flutter. Circulation 2000; 101: $2178-2184$.

12. Costa AD, Faure E, Thevenin J, Messier M, Bernard S, Abdel K, et al. Effect of isthmus anatomy and ablation catheter on radiofrequency catheter ablation of the cavotricuspid isthmus. Circulation 2004; 110: $1030-1035$.

13. Ohba Y, Shimoike E, Ueda N, Maruyama T, Kaji Y, Fujino T, et al. Influence of right atrial structure on outcome of radiofrequency catheter ablation for common atrial flutter. Jpn Circ J 2000; 64: 741-744.

14. Ouali S, Anselme F, Savoure A, Cribier A. Acute coronary occlusion during radiofrequency catheter ablation of typical atrial flutter. J Cardiovasc Electrophysiol 2002; 13: 1047 - 1049. 